\title{
Identification and Biochemical Characterization of some Strains of Clavibacter michiganensis subsp. Michiganensis, in Romania
}

\author{
Mariana-Simona STANCU* and Rodi MITREA \\ University of Craiova, Faculty of Horticulture, A.I. Cuza, no. 13, Craiova, Romania \\ *corresponding author: simosebabia@gmail.com
}

BulletinUASVM Horticulture 77(2) / 2020

Print ISSN 1843-5254, Electronic ISSN 1843-5394

DOI:10.15835/buasvmcn-hort: 2020.0027

\begin{abstract}
Clavibacter michiganensis subsp. michiganensis is responsible for the 'bacterial canker of tomato'. The study aimed to identify and characterize biochemically some strains of the bacteria. Five strains were used for biochemical identification and characterization and two reference strains were used as positive controls. The biochemical characterization was performed with Biolog Microbial Identification System. All the strains used many carbon sources and were positive for growth in the presence of: $1 \% \mathrm{NaCl}$, Nalidixic Acid, Aztreonam, Potassium Tellurite and $\mathrm{pH} \mathrm{6}$, but negative for growth and sensitivite to: Troleandomycin, Lincomycin, Vancomycin, Fusidic Acid, Eimfamycin SV, Guanidine HCl, D-Serine, Minocycline and Niaproof 4. The differences between the Biolog system database and the studied strains did not change their classification at the level of genus and species, but modifed or did not allow the classification at the subspecies level. Furthermore, the analyzed strains had metabolic diversity.
\end{abstract}

Keywords: Biolog system, spectrophotometry, optic

\section{Introduction}

At the beginning of the $20^{\text {th }}$ century, in 1910 , Smith discovered in the state of Michigan, United States of America, a harmful bacteria that causes the wilting of tomato plants (Singh and Bharat, 2017). In Romania, it was reported by Elena Bucur, since 1955 (Severin and Iliescu, 2006).

It is a Gram positive bacteria, aerobic, nonmobile, solitary or in pairs, capsulated, slightly curved rod-shaped, sometimes with swellings or branches at some ends (Severin and Iliescu, 2006). Optimal growth and development conditions of Clavibacter michiganensis subsp. michiganensis are temperatures between 20 and $30^{\circ} \mathrm{C}$ and $\mathrm{pH} 7$ 8 (Sen et al., 2015).
Clavibactermichiganensissubsp.michiganensis produces very different symptoms which depend on: the type of infection (localized or systemic), the sensitivity of the cultivar, the age of the plant, the virulence of the pathogen, the nutritional status of the host plant and the environmental conditions (humidity and temperature).

The main host plant of economic importance is Solanum lycopersicum, but this bacteria also attacks other sensitive species of Solanum spp. (https://gd.eppo.int/taxon/CORBMI/hosts). Other plants of the Solanaceae family can naturally be infected (Capsicum sativum, Capsicum annuum, Capsicum frutescens and Solanum melongena). 
Solanaceous and non-solanaceous weeds can spread the bacteria being natural reservoirs of infection.

Mainly, Clavibacter michiganensis subsp. michiganensis can be transmitted with the planting material (seedlings and seeds). Secondary, it spreads with: infected debris and soil, infected host plants with or without symptoms, equipment and other contaminated materials and agricultural practices (https://wiki.bugwood.org/Clavibacter_ michiganensis_subsp._michiganensis).

According to the literature there are three mechanisms that could explain the wilting of plants in case of infection with Clavibacter michiganensis subsp. michiganensis namely: mechanical obstruction of xylem due to colonization of vessels with bacteria, toxic action of exopolysaccharides and enzymatic attack on plant tissues (Jahr et al., 2000).

Studies show that strains of Clavibacter michiganensis subsp. michiganensis are different morphologically, biochemically, phenotypically and genetically.The present study aims to identify and characterize biochemically some strains of the bacteria from different counties of Romania.

\section{Materials and Methods}

Five strains of Clavibacter michiganensis subsp. michiganensis, from three counties (Argeș, Suceava and Satu Mare) of our country were used for biochemical identification and characterization. The strains $\mathrm{Cmm} 19-3857, \mathrm{Cmm}$ 19-3861 and $\mathrm{Cmm}$ 19-4326 were isolated from Solanum lycopersicum cv. 'Colibri', the strain $\mathrm{Cmm}$ 19-4327 from S. lycopersicum cv. 'Kyveli' and the strain Cmm 19-4819 from S. lycopersicum cv 'Inima de bou'.

As positive controls were used two reference strains of Clavibacter michiganensis subsp. michiganensis (NCPPB 2979 and PD 223) both originating in Hungary, one purchased from the United Kingdom (Food and Environment Research Agency Sand Hutton) and the other from the Netherlands (Plant Protection Service). All strains analyzed were cryopreserved at $-80^{\circ} \mathrm{C}$ using the Protect $^{\mathrm{TM}}$ system.

The biochemical characterization was performed with 'Biolog's Microbial Identification System' which determines a phenotipic fingerprint of the strains of Clavibacter michiganensis subsp. michiganensis. This system uses MicroPlates with
94 phenotypic tests: 71 carbon sources and 23 chemical sensitivity tests. Microorganisms use or not use different carbon sources from MicroPlate wells. Where the carbon source is used, a redox reaction which reduces tetrazole occures and due to cellular respiration the well is colored in purple. Where the microorganism does not utilize the carbon source no reaction occurs and the wells remain colorless. The pattern of colored and noncolored wells is specific to each strain (User guide - Identification system Biolog).

Four steps were required to perform this test: isolating the culture, preparing a bacterial suspension in an inoculation fluid with a certain density, incubating the GenIII microplates at a certain temperature, reading and interpreting the results. Thus, 1-2 balls loaded with bacterial cells from each strain of Clavibacter michiganensis subsp. michiganensis were placed on culture media BUG (Biolog Universal Growth, special media for this test prepared according to the manufacturer's recommendations) or on nonselective media (Yeast extract Peptone Glucose Agar or Nutrient Agar). Isolated Petri dishes were incubated, at $27^{\circ} \mathrm{C}$, between 24 and 48 hours, until the first bacterial growths appeared. Extended incubation (for more than 48 hours) was avoided, because the bacteria had to be viable and it had to have metabolic activity, in order to react with the different carbon sources on the Gen III microplates (96 wells). After the first growths appeared, a bacterial colony was taken with a loopful and introduced into an inoculation fluid (IF-A) specific to aerobic bacteria. The fluid tubes were agitated by reversing until the colonies were dispersed in the liquid media. Then, using a turbidimeter, the cell density (transmittance) of the vials with the inoculation fluid was measured. If the transmittance of the samples was between $92-98 \%$, the fluid was pipetted onto a Gen III microplate. If the fluid transmittance was below $92 \%$, it is diluted by adding IF-A, until it reaches the desired value. If the transmittance was over 98\%, biological material (colonies) is added, until it reaches the desired value. Then the Gen III microplates (Fig. 1) were loaded with $100 \mu \mathrm{l}$ of inoculation fluid per well and incubated at $30^{\circ} \mathrm{C}$, for 22 hours, in an OmniLog incubator. 


\begin{tabular}{|c|c|c|c|c|c|c|c|c|c|c|c|}
\hline $\begin{array}{c}\text { Al } \\
\text { Negative } \\
\text { Control }\end{array}$ & $\begin{array}{c}\text { A2 } \\
\text { Dextrin }\end{array}$ & $\begin{array}{c}\text { A3 } \\
\text { D-Maltose }\end{array}$ & $\begin{array}{c}\text { A4 } \\
\text { D-Trehalose }\end{array}$ & $\begin{array}{c}\text { A5 } \\
\text { D- } \\
\text { Cellobiose }\end{array}$ & $\begin{array}{c}\text { A6 } \\
\text { Gentiobiose }\end{array}$ & $\begin{array}{c}\text { A7 } \\
\text { Sucrose }\end{array}$ & $\begin{array}{c}\text { A8 } \\
\text { D-Turanose }\end{array}$ & $\begin{array}{c}\text { A9 } \\
\text { Stachyose }\end{array}$ & $\begin{array}{c}\text { A10 } \\
\text { Positive } \\
\text { Control }\end{array}$ & $\begin{array}{l}\text { A11 } \\
\text { pH } 6\end{array}$ & $\begin{array}{l}\mathrm{A} 12 \\
\mathrm{pH} 5\end{array}$ \\
\hline $\begin{array}{c}\text { B1 } \\
\text { D-Raffinose }\end{array}$ & $\begin{array}{c}\text { B2 } \\
\alpha \text {-D-Lactose }\end{array}$ & $\begin{array}{c}\text { B3 } \\
\text { D-Melibiose }\end{array}$ & $\begin{array}{c}\text { B4 } \\
\text { B-Methyl-D- } \\
\text { Glucoside }\end{array}$ & $\begin{array}{c}\text { B5 } \\
\text { D-Salicin }\end{array}$ & $\begin{array}{c}\text { B6 } \\
\text { N-Acetyl-D- } \\
\text { Glucosamine }\end{array}$ & $\begin{array}{c}\text { B7 } \\
\text { N-Acetyl- } \beta \text {-D- } \\
\text { Mannosamine }\end{array}$ & $\begin{array}{c}\text { B8 } \\
\text { N-Acetyl-D- } \\
\text { Galactosamine }\end{array}$ & $\begin{array}{c}\text { B9 } \\
\text { N-Acetyl } \\
\text { Neuramini } \\
\text { c Acid } \\
\end{array}$ & $\begin{array}{c}\mathrm{B} 10 \\
1 \% \mathrm{NaCl}\end{array}$ & $\begin{array}{c}\mathrm{B} 11 \\
4 \% \mathrm{NaCl}\end{array}$ & $\begin{array}{c}\mathrm{B} 12 \\
8 \% \mathrm{NaCl}\end{array}$ \\
\hline $\begin{array}{c}\text { Cl } \\
\alpha \text {-D-Glucose }\end{array}$ & $\begin{array}{c}\text { C2 } \\
\text { D-Mannose }\end{array}$ & $\begin{array}{c}\text { C3 } \\
\text { D-Fructose }\end{array}$ & $\begin{array}{c}\text { C4 } \\
\text { D-Galactose }\end{array}$ & $\begin{array}{c}\text { C5 } \\
\text { 3-Methyl } \\
\text { Glucose } \\
\end{array}$ & $\begin{array}{c}\text { C6 } \\
\text { D-Fucose }\end{array}$ & $\begin{array}{c}\text { C7 } \\
\text { L-Fucose }\end{array}$ & $\begin{array}{c}\text { C8 } \\
\text { L-Rhamnose }\end{array}$ & $\begin{array}{c}\mathrm{C} 9 \\
\text { Inosine }\end{array}$ & $\begin{array}{c}\text { C10 } \\
\text { 1\%Sodium } \\
\text { Lactate } \\
\end{array}$ & $\begin{array}{c}\text { C11 } \\
\text { Fusidic Acid }\end{array}$ & $\begin{array}{c}\text { C12 } \\
\text { D-Serine }\end{array}$ \\
\hline $\begin{array}{c}\text { D1 } \\
\text { D-Sorbitol }\end{array}$ & $\begin{array}{c}\text { D2 } \\
\text { D-Mannitol }\end{array}$ & $\begin{array}{c}\text { D3 } \\
\text { D-Arabitol }\end{array}$ & $\begin{array}{c}\text { D4 } \\
\text { myo-Inositol }\end{array}$ & $\begin{array}{c}\text { D5 } \\
\text { Glycerol }\end{array}$ & $\begin{array}{c}\text { D6 } \\
\text { D-Glucose-6- } \\
\text { PO4 } \\
\end{array}$ & $\begin{array}{c}\text { D7 } \\
\text { D-Fructose-6- } \\
\text { PO4 } \\
\end{array}$ & $\begin{array}{c}\text { D8 } \\
\text { D-Aspartic Acid }\end{array}$ & $\begin{array}{c}\text { D9 } \\
\text { D-Serine }\end{array}$ & $\begin{array}{c}\text { D10 } \\
\text { Troleandomyc } \\
\text { in } \\
\end{array}$ & $\begin{array}{c}\text { D11 } \\
\text { Rimfamycin } \\
\text { SV } \\
\end{array}$ & $\begin{array}{c}\text { D12 } \\
\text { Minocycline }\end{array}$ \\
\hline $\begin{array}{c}\text { E1 } \\
\text { Gelatin }\end{array}$ & $\begin{array}{c}\text { E2 } \\
\text { Glycyl-L- } \\
\text { Proline } \\
\end{array}$ & $\begin{array}{c}\text { E3 } \\
\text { L-Alanine }\end{array}$ & $\begin{array}{c}\text { E4 } \\
\text { L-Arginine }\end{array}$ & $\begin{array}{c}\text { E5 } \\
\text { L-Aspartic } \\
\text { Acid } \\
\end{array}$ & $\begin{array}{c}\text { E6 } \\
\text { L-Glutamic Acid }\end{array}$ & $\begin{array}{c}\text { E7 } \\
\text { L-Histidine }\end{array}$ & $\begin{array}{c}\text { E8 } \\
\text { L-Pyroglutamic } \\
\text { Acid } \\
\end{array}$ & $\begin{array}{c}\text { E9 } \\
\text { L-Serine }\end{array}$ & $\begin{array}{c}\text { E10 } \\
\text { Lincomycin }\end{array}$ & $\begin{array}{c}\text { E11 } \\
\text { Guanidine } \\
\mathrm{HCl} \\
\end{array}$ & $\begin{array}{c}\text { E12 } \\
\text { Niaproof } 4\end{array}$ \\
\hline $\begin{array}{c}\mathrm{F} 1 \\
\text { Pectin }\end{array}$ & $\begin{array}{c}\text { F2 } \\
\text { D- } \\
\text { Galacturonic } \\
\text { Acid }\end{array}$ & $\begin{array}{c}\text { F3 } \\
\text { L-Galactonic } \\
\text { Acid } \\
\text { Lactone }\end{array}$ & $\begin{array}{c}\text { F4 } \\
\text { D-Gluconic } \\
\text { Acid }\end{array}$ & $\begin{array}{c}\text { F5 } \\
\text { D- } \\
\text { Glucuronic } \\
\text { Acid }\end{array}$ & $\begin{array}{c}\text { F6 } \\
\text { Glucuronamide }\end{array}$ & $\begin{array}{c}\text { F7 } \\
\text { Mucic Acid }\end{array}$ & $\begin{array}{c}\text { F8 } \\
\text { Quinic Acid }\end{array}$ & $\begin{array}{c}\text { F9 } \\
\text { D- } \\
\text { Saccharic } \\
\text { Acid }\end{array}$ & $\begin{array}{c}\text { F10 } \\
\text { Vancomycin }\end{array}$ & $\begin{array}{c}\text { F11 } \\
\text { Tetrazolium } \\
\text { Violet }\end{array}$ & $\begin{array}{c}\text { F12 } \\
\text { Tetrazolium } \\
\text { Blue }\end{array}$ \\
\hline $\begin{array}{c}\text { Gl } \\
\text { p-Hydroxy- } \\
\text { Phenylacetic } \\
\text { Acid }\end{array}$ & $\begin{array}{c}\text { G2 } \\
\text { Methyl } \\
\text { Pyruvate }\end{array}$ & $\begin{array}{c}\text { G3 } \\
\text { D-Lactic } \\
\text { Acid Methyl } \\
\text { Ester } \\
\end{array}$ & $\begin{array}{c}\text { G4 } \\
\text { L-Lactic } \\
\text { Acid }\end{array}$ & $\begin{array}{c}\text { G5 } \\
\text { Citric Acid }\end{array}$ & $\begin{array}{c}\text { G6 } \\
\alpha \text {-Keto-Glutaric } \\
\text { Acid }\end{array}$ & $\begin{array}{c}\text { G7 } \\
\text { D-Malic Acid }\end{array}$ & $\begin{array}{c}\text { G8 } \\
\text { L-Malic Acid }\end{array}$ & $\begin{array}{c}\text { G9 } \\
\text { Bromo- } \\
\text { Succinic } \\
\text { Acid }\end{array}$ & $\begin{array}{c}\text { G10 } \\
\text { Nalidixic Acid }\end{array}$ & $\begin{array}{c}\text { G11 } \\
\text { Lithium } \\
\text { Chloride }\end{array}$ & $\begin{array}{c}\text { G12 } \\
\text { Potassium } \\
\text { Tellurite }\end{array}$ \\
\hline $\begin{array}{c}\mathrm{Hl} \\
\text { Tween } 40\end{array}$ & $\begin{array}{c}\text { H2 } \\
\gamma \text {-Anino- } \\
\text { Butryric } \\
\text { Acid }\end{array}$ & $\begin{array}{c}\text { H3 } \\
\alpha \text {-Hydroxy } \\
\text { Butyric Acid }\end{array}$ & $\begin{array}{c}\mathrm{H} 4 \\
\beta \text {-Hydroxy- } \\
\text { D,L-Butyric } \\
\text { Acid }\end{array}$ & $\begin{array}{c}\text { H5 } \\
\alpha \text {-Keto- } \\
\text { Butyric Acid }\end{array}$ & $\begin{array}{c}\mathrm{H} 6 \\
\text { Acetoacetic Acid }\end{array}$ & $\begin{array}{c}\mathrm{H} 7 \\
\text { Propionic Acid }\end{array}$ & $\begin{array}{c}\mathrm{H} 8 \\
\text { Acetic Acid }\end{array}$ & $\begin{array}{c}\mathrm{H} 9 \\
\text { Formic } \\
\text { Acid }\end{array}$ & $\begin{array}{c}\text { H10 } \\
\text { Aztreonam }\end{array}$ & $\begin{array}{c}\text { H11 } \\
\text { Sodium } \\
\text { Butyrate }\end{array}$ & $\begin{array}{c}\text { H12 } \\
\text { Sodium } \\
\text { Bromate }\end{array}$ \\
\hline
\end{tabular}

Figure 1. MicroPlates GEN III (User guide - Identification system Biolog)

\section{Results and discussions}

After the end of the incubation period, there were performed three types of readings: visual, optical and spectrophotometric.

Visual readings of the microplates established that in some wells the redox reaction took place, therefore the bacteria of Clavibacter michiganensis subsp. michiganensis $(\mathrm{Cmm})$ were metabolically active, breathed and reduced tetrazole, coloring the well into lilac (Fig. 2).

Optical readings were made at the Omnilog reader (Biolog equipment), provided with a video camera that takes the images, at certain times. The readings were performed for 22 hours, from half to half an hour. During this period the bacterial strains were biochemically characterized and identified,

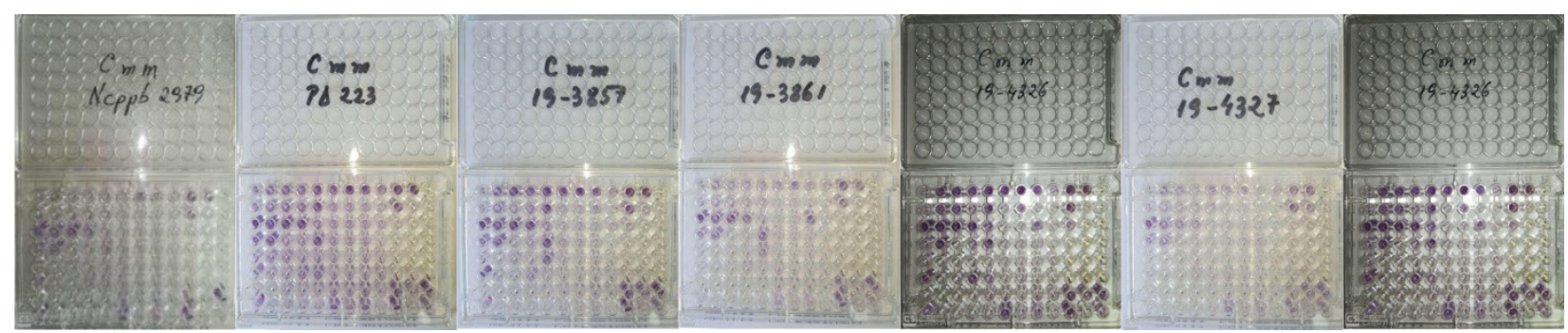

Figure 2. Color reactions - GEN III MicroPlates contaminated with live cells of $\mathrm{Cmm}$ 


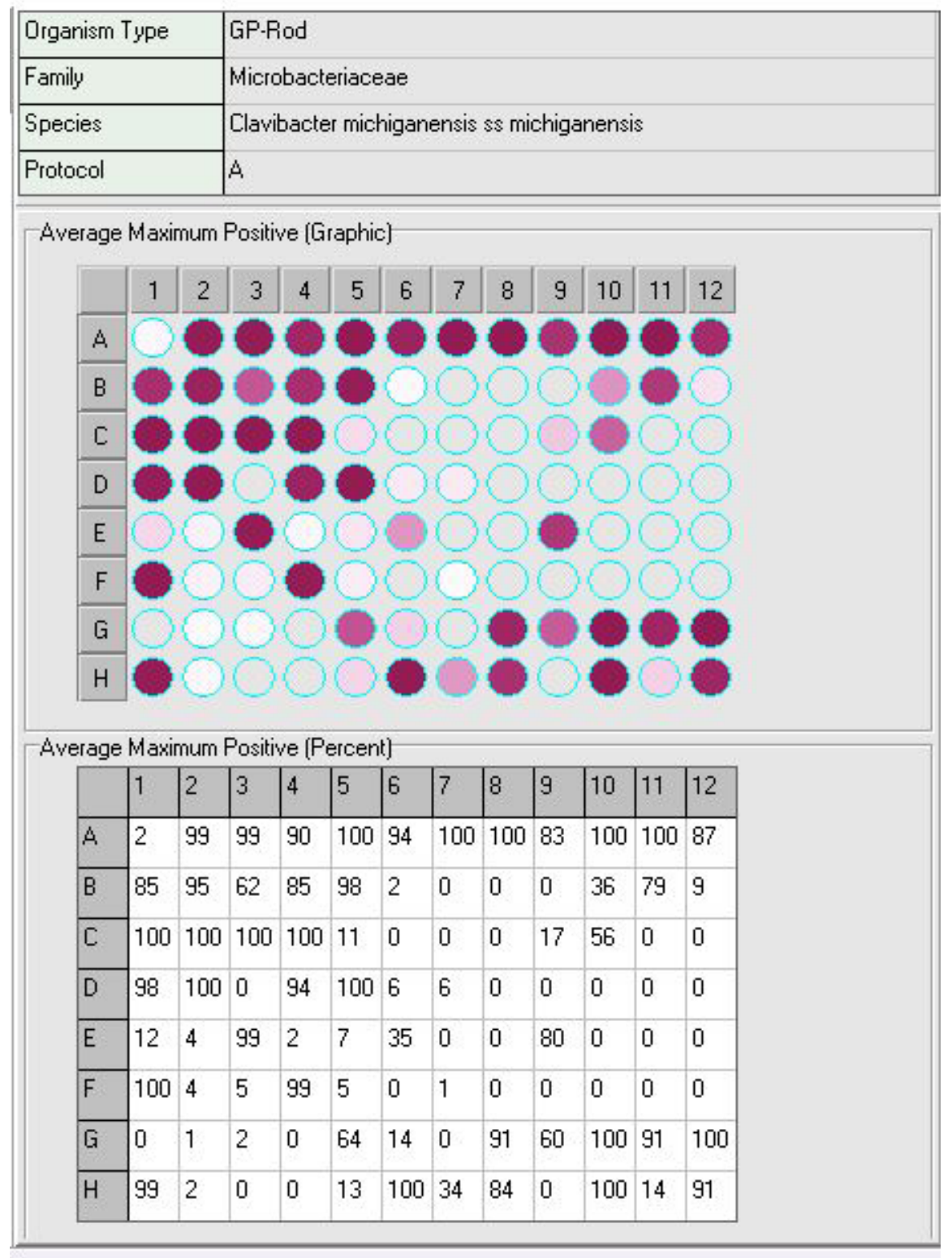

Figure 3. Phenotipic profile of $\mathrm{Cmm}$ (User guide - Identification system Biolog)

by comparing their metabolic fingerprint with a database with over 2000 profiles, for Grampositive and Gram-negative microorganisms.

Spectrophotometric readings were performed at MicroStationTM reader (Biolog equipment) at two densities (absorbance) $590 \mathrm{~nm}$ and $750 \mathrm{~nm}$.

In the database of the Biolog system (GEN III) there is the metabolic profile of a strain of Clavibacter michiganensis subsp. michiganensis (Fig. 3). The interpretation of the reactions of this strain is shown in Figure 4. The strains taken in the study were characterized by comparison with this metabolic profile.
Columns 1-9 were used for carbon source utilization tests. All reactions in these columns are compared to the reaction in well A1, which is the negative control well. Therefore, the reactions in the wells in columns 1-9 which give a color similar to well A1 (the absence of any color) are considered negative because they do not use the respective carbon source. The wells that have been colored in lilac (more intense or weaker) are considered positive because they metabolize the respective substrate, using the carbon source.

Columns 10-12 were used for chemical sensitivity tests. The reactions in these wells are com- 


\begin{tabular}{|c|c|c|c|c|c|c|c|c|c|c|c|}
\hline $\begin{array}{c}\text { Al } \\
\text { Negative } \\
\text { Control } \\
-\end{array}$ & $\begin{array}{c}\text { A2 } \\
\text { Dextrin } \\
+\end{array}$ & $\begin{array}{c}\text { A3 } \\
\text { D-Maltose } \\
+\end{array}$ & $\begin{array}{c}\text { A4 } \\
\text { D-Trehalose } \\
+\end{array}$ & $\begin{array}{c}\text { A5 } \\
\text { D-Cellobiose } \\
+\end{array}$ & $\begin{array}{c}\text { A6 } \\
\text { Gentiobiose } \\
+\end{array}$ & $\begin{array}{c}\text { A7 } \\
\text { Sucrose } \\
+\end{array}$ & $\begin{array}{c}\text { A8 } \\
\text { D-Turanose } \\
+\end{array}$ & $\begin{array}{c}\text { A9 } \\
\text { Stachyose } \\
+\end{array}$ & $\begin{array}{l}\text { A10 } \\
\text { Positive } \\
\text { Control } \\
+\end{array}$ & $\begin{array}{c}\text { All } \\
\mathrm{pH} 6 \\
+\end{array}$ & $\begin{array}{c}\mathrm{A} 12 \\
\mathrm{pH} 5 \\
+\end{array}$ \\
\hline $\begin{array}{c}\text { B1 } \\
\text { D-Raffinose } \\
+\end{array}$ & $\begin{array}{c}\text { B2 } \\
\alpha \text {-D-Lactose } \\
+\end{array}$ & $\begin{array}{c}\text { B3 } \\
\text { D-Melibiose } \\
+\end{array}$ & $\begin{array}{c}\text { B4 } \\
\text { B-Methyl-D- } \\
\text { Glucoside } \\
+\end{array}$ & $\begin{array}{c}\text { B5 } \\
\text { D-Salicin } \\
+\end{array}$ & $\begin{array}{c}\text { B6 } \\
\text { N-Acetyl-D- } \\
\text { Glucosamine } \\
-\end{array}$ & $\begin{array}{c}\text { B7 } \\
\text { N-Acetyl- } \beta-D- \\
\text { Mannosamine } \\
-\end{array}$ & $\begin{array}{c}\text { B8 } \\
\text { N-Acetyl-D- } \\
\text { Galactosamine } \\
-\end{array}$ & $\begin{array}{c}\text { B9 } \\
\text { N-Acetyl } \\
\text { Neuraminic } \\
\text { Acid } \\
-\end{array}$ & $\begin{array}{c}\mathrm{B} 10 \\
1 \% \mathrm{NaCl} \\
+\end{array}$ & $\begin{array}{c}\mathrm{B} 11 \\
4 \% \mathrm{NaCl} \\
+\end{array}$ & $\begin{array}{c}\mathrm{B} 12 \\
8 \% \mathrm{NaCl} \\
+ \\
\text { (slab) }\end{array}$ \\
\hline $\begin{array}{c}\mathrm{Cl} \\
\alpha \text {-D-Glucose } \\
+\end{array}$ & $\begin{array}{c}\text { C2 } \\
\text { D-Mannose } \\
+\end{array}$ & $\begin{array}{c}\text { C3 } \\
\text { D-Fructose } \\
+\end{array}$ & $\begin{array}{c}\text { C4 } \\
\text { D-Galactose } \\
+\end{array}$ & $\begin{array}{c}\text { C5 } \\
\text { 3-Methyl } \\
\text { Glucose } \\
+ \\
\text { (slab) }\end{array}$ & $\begin{array}{c}\text { C6 } \\
\text { D-Fucose } \\
-\end{array}$ & $\begin{array}{c}\text { C7 } \\
\text { L-Fucose } \\
-\end{array}$ & $\begin{array}{c}\text { C8 } \\
\text { L-Rhamnose } \\
-\end{array}$ & $\begin{array}{c}\text { C9 } \\
\text { Inosine } \\
+ \text { (slab) }\end{array}$ & $\begin{array}{c}\mathrm{C} 10 \\
\text { 1\% Sodium } \\
\text { Lactate } \\
+\end{array}$ & $\begin{array}{c}\text { C11 } \\
\text { Fusidic Acid } \\
\text { - }\end{array}$ & $\begin{array}{c}\text { C12 } \\
\text { D-Serine } \\
-\end{array}$ \\
\hline $\begin{array}{c}\text { D1 } \\
\text { D-Sorbitol } \\
+\end{array}$ & $\begin{array}{c}\text { D2 } \\
\text { D-Mannitol } \\
+\end{array}$ & $\begin{array}{c}\text { D3 } \\
\text { D-Arabitol } \\
-\end{array}$ & $\begin{array}{c}\text { D4 } \\
\text { myo-Inositol } \\
+\end{array}$ & $\begin{array}{c}\text { D5 } \\
\text { Glycerol } \\
+\end{array}$ & $\begin{array}{c}\text { D6 } \\
\text { D-Glucose-6- } \\
\text { PO4 } \\
\text { +(slab) }\end{array}$ & $\begin{array}{c}\text { D7 } \\
\text { D-Fructose-6- } \\
\text { PO4 } \\
+ \text { (slab) }\end{array}$ & $\begin{array}{c}\text { D8 } \\
\text { D-Aspartic } \\
\text { Acid } \\
-\end{array}$ & $\begin{array}{c}\text { D9 } \\
\text { D-Serine } \\
-\end{array}$ & $\begin{array}{c}\text { D10 } \\
\text { Troleandomyc } \\
\text { in } \\
-\end{array}$ & $\begin{array}{c}\text { D11 } \\
\text { Rimfamycin } \\
\text { SV } \\
-\end{array}$ & $\begin{array}{c}\text { D12 } \\
\text { Minocycline } \\
-\end{array}$ \\
\hline $\begin{array}{c}\text { E1 } \\
\text { Gelatin } \\
+ \\
\text { (slab) }\end{array}$ & $\begin{array}{c}\text { E2 } \\
\text { Glycyl-L- } \\
\text { Proline } \\
-\end{array}$ & $\begin{array}{c}\text { E3 } \\
\text { L-Alanine } \\
+\end{array}$ & $\begin{array}{c}\text { E4 } \\
\text { L-Arginine } \\
-\end{array}$ & $\begin{array}{c}\text { E5 } \\
\text { L-Aspartic } \\
\text { Acid } \\
+ \\
\text { (slab) }\end{array}$ & $\begin{array}{c}\text { E6 } \\
\text { L-Glutamic } \\
\text { Acid } \\
+\end{array}$ & $\begin{array}{c}\text { E7 } \\
\text { L-Histidine } \\
-\end{array}$ & \begin{tabular}{|c|} 
E8 \\
L- \\
Pyroglutamic \\
Acid \\
-
\end{tabular} & $\begin{array}{c}\text { E9 } \\
\text { L-Serine } \\
+\end{array}$ & $\begin{array}{c}\text { E10 } \\
\text { Lincomycin } \\
-\end{array}$ & $\begin{array}{c}\text { E11 } \\
\text { Guanidine } \mathrm{HCl} \\
-\end{array}$ & $\begin{array}{c}\text { E12 } \\
\text { Niaproof } 4 \\
-\end{array}$ \\
\hline $\begin{array}{c}\text { Fl } \\
\text { Pectin } \\
+\end{array}$ & $\begin{array}{c}\text { F2 } \\
\text { D-Galacturonic } \\
\text { Acid } \\
-\end{array}$ & $\begin{array}{c}\text { F3 } \\
\text { L-Galactonic } \\
\text { Acid Lactone } \\
-\end{array}$ & $\begin{array}{c}\text { F4 } \\
\text { D-Gluconic } \\
\text { Acid } \\
+\end{array}$ & $\begin{array}{c}\text { F5 } \\
\text { D-Glucuronic } \\
\text { Acid } \\
-\end{array}$ & $\begin{array}{c}\text { F6 } \\
\text { Glucuronamid } \\
\mathrm{e} \\
-\end{array}$ & $\begin{array}{c}\text { F7 } \\
\text { Mucic Acid } \\
-\end{array}$ & $\begin{array}{c}\text { F8 } \\
\text { Quinic Acid } \\
-\end{array}$ & $\begin{array}{c}\text { F9 } \\
\text { D-Saccharic } \\
\text { Acid } \\
-\end{array}$ & $\begin{array}{c}\text { F10 } \\
\text { Vancomycin } \\
-\end{array}$ & $\begin{array}{c}\text { F11 } \\
\text { Tetrazolium } \\
\text { Violet } \\
-\end{array}$ & $\begin{array}{c}\text { F12 } \\
\text { Tetrazolium } \\
\text { Blue } \\
-\end{array}$ \\
\hline $\begin{array}{c}\text { G1 } \\
\text { p-Hydroxy- } \\
\text { Phenylacetic } \\
\text { Acid } \\
-\end{array}$ & $\begin{array}{c}\text { G2 } \\
\text { Methyl } \\
\text { Pyruvate } \\
-\end{array}$ & $\begin{array}{c}\text { G3 } \\
\text { D-Lactic Acid } \\
\text { Methyl Ester } \\
-\end{array}$ & $\begin{array}{c}\text { G4 } \\
\text { L-Lactic Acid } \\
-\end{array}$ & $\begin{array}{c}\text { G5 } \\
\text { Citric Acid } \\
+\end{array}$ & $\begin{array}{c}\text { G6 } \\
\alpha \text {-Keto- } \\
\text { Glutaric Acid } \\
+ \text { (slab) }\end{array}$ & $\begin{array}{c}\text { G7 } \\
\text { D-Malic Acid } \\
-\end{array}$ & $\begin{array}{c}\text { G8 } \\
\text { L-Malic Acid } \\
+\end{array}$ & $\begin{array}{c}\text { G9 } \\
\text { Bromo- } \\
\text { Succinic Acid } \\
+\end{array}$ & $\begin{array}{c}\text { G10 } \\
\text { Nalidixic } \\
\text { Acid } \\
+\end{array}$ & $\begin{array}{c}\text { G1l } \\
\text { Lithium } \\
\text { Chloride } \\
+\end{array}$ & $\begin{array}{c}\text { G12 } \\
\text { Potassium } \\
\text { Tellurite } \\
+\end{array}$ \\
\hline $\begin{array}{c}\text { H1 } \\
\text { Tween } 40 \\
+\end{array}$ & $\begin{array}{c}\mathrm{H} 2 \\
\gamma \text {-Anino- } \\
\text { Butryric Acid } \\
-\end{array}$ & $\begin{array}{c}\mathrm{H} 3 \\
\alpha \text {-Hydroxy } \\
\text { Butyric Acid } \\
-\end{array}$ & $\begin{array}{c}\text { H4 } \\
\text { B-Hydroxy- } \\
\text { D,L-Butyric } \\
\text { Acid } \\
-\end{array}$ & $\begin{array}{c}\text { H5 } \\
\alpha \text {-Keto-Butyric } \\
\text { Acid } \\
+ \\
\text { (slab) }\end{array}$ & $\begin{array}{c}\text { H6 } \\
\text { Acetoacetic } \\
\text { Acid } \\
+\end{array}$ & $\begin{array}{c}\text { H7 } \\
\text { Propionic Acid } \\
+\end{array}$ & $\begin{array}{c}\text { H8 } \\
\text { Acetic Acid } \\
+\end{array}$ & $\begin{array}{c}\text { H9 } \\
\text { Formic Acid } \\
-\end{array}$ & $\begin{array}{c}\text { H10 } \\
\text { Aztreonam } \\
+\end{array}$ & $\begin{array}{c}\text { H11 } \\
\text { Sodium } \\
\text { Butyrate } \\
+ \\
\text { (slab) }\end{array}$ & $\begin{array}{l}\text { H12 } \\
\text { Sodium } \\
\text { Bromate } \\
+\end{array}$ \\
\hline
\end{tabular}

Figure 4. Interpretation of the phenotypic profile of strain of $\mathrm{Cmm}$ $+=$ positive reaction; - = negative reaction

\begin{tabular}{|c|c|c|c|c|c|c|c|c|c|c|c|}
\hline 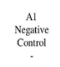 & 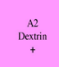 & 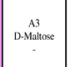 & orither & ocks & cats & $\ldots$ & 隹 & 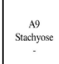 & 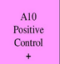 & 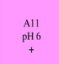 & ${ }_{\substack{A 12 \\
\text { Hits }}}$ \\
\hline 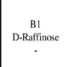 & 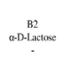 & 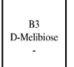 & 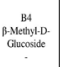 & \begin{tabular}{|l|} 
Bst \\
Dessicinin
\end{tabular} & 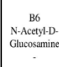 & 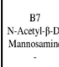 & 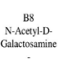 & 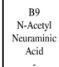 & 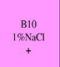 & 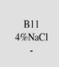 & 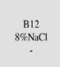 \\
\hline encicher & 票 & 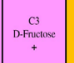 & 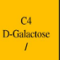 & 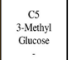 & 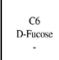 & Lencues & 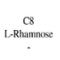 & 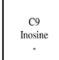 & 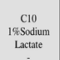 & $\begin{array}{c}\text { cill } \\
\text { rusulex }\end{array}$ & 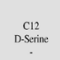 \\
\hline 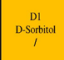 & 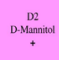 & 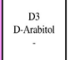 & 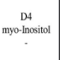 & 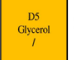 & 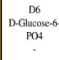 & 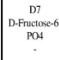 & 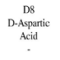 & 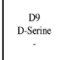 & 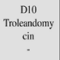 & 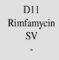 & 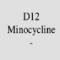 \\
\hline 良战 & 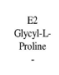 & 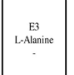 & $\begin{array}{l}\text { Lef } \\
\text { Lafyice }\end{array}$ & 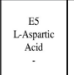 & 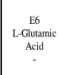 & 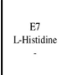 & 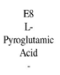 & 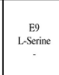 & 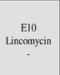 & Bil & 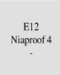 \\
\hline 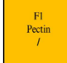 & 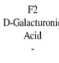 & 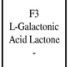 & 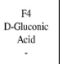 & 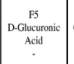 & 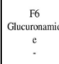 & 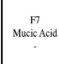 & 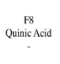 & 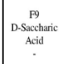 & 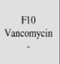 & 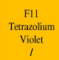 & 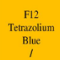 \\
\hline 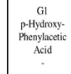 & 必 & 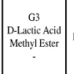 & 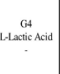 & $\begin{array}{c}\text { cisioxide } \\
\text { cint }\end{array}$ & 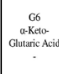 & 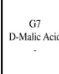 & ist & 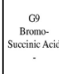 & 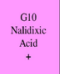 & 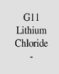 & 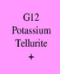 \\
\hline 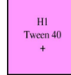 & 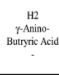 & 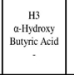 & 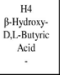 & 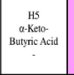 & 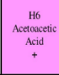 & 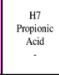 & 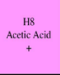 & 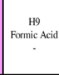 & 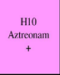 & 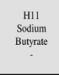 & 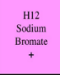 \\
\hline
\end{tabular}

\begin{tabular}{|c|c|c|c|c|c|c|c|c|c|c|c|}
\hline 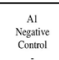 & 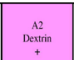 & 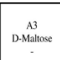 & 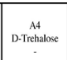 & 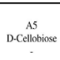 & 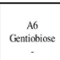 & 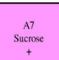 & 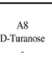 & 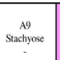 & 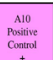 & , & 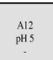 \\
\hline 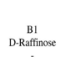 & oblumex & 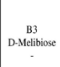 & 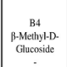 & 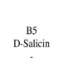 & 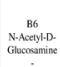 & 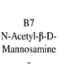 & 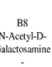 & 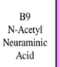 & 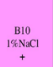 & 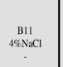 & 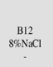 \\
\hline ocinume & Dicmose & 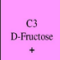 & ocis & 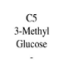 & 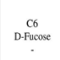 & 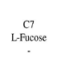 & LResingex & 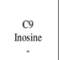 & 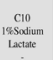 & 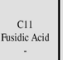 & 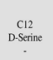 \\
\hline oftetid & 告 & 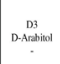 & 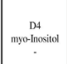 & 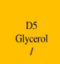 & 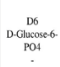 & 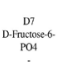 & 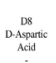 & 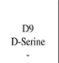 & 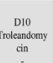 & 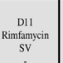 & 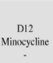 \\
\hline 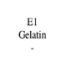 & 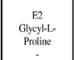 & $\begin{array}{c}\text { E } \\
\text { Lhalume }\end{array}$ & 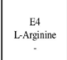 & 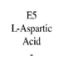 & 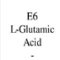 & 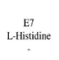 & 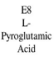 & 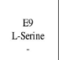 & 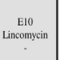 & call & 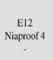 \\
\hline 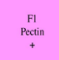 & 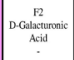 & 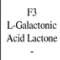 & 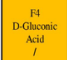 & 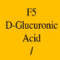 & 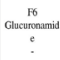 & 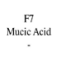 & 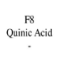 & 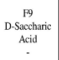 & vane & 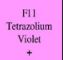 & 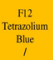 \\
\hline 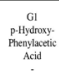 & 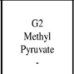 & 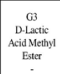 & 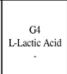 & 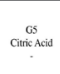 & 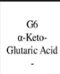 & Disulectasid & 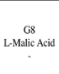 & 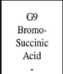 & 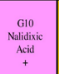 & 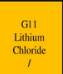 & 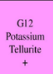 \\
\hline 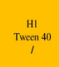 & 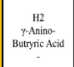 & 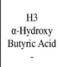 & 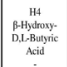 & 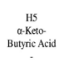 & 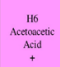 & 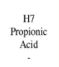 & 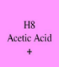 & 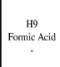 & 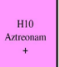 & 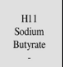 & 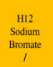 \\
\hline
\end{tabular}

Figure 5. Interpretation of metabolic profile Cmm Ncppb 2979

optical reading (left), spectrophotometric reading (right)

+ = positive reaction, lilac color; - = negative reaction, no color; / = doubtful reaction, yellow color 


\begin{tabular}{|c|c|c|c|c|c|c|c|c|c|c|c|}
\hline 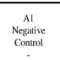 & $\underset{\substack{A 2 \\
\text { Deutrin } \\
+}}{2}$ & D.MAlase & 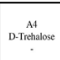 & Dallotione & 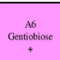 & 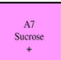 & $\begin{array}{c}\text { As } \\
\text { Dhrinaese } \\
+\end{array}$ & Shatipese & 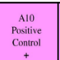 & 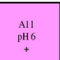 & 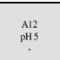 \\
\hline 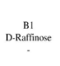 & 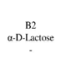 & 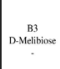 & 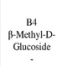 & $\begin{array}{c}\text { Bs } \\
\text { D.silitin }\end{array}$ & 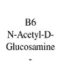 & 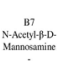 & 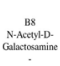 & 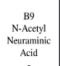 & 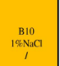 & 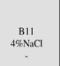 & $\begin{array}{l}\text { siz } \\
\text { sativac }\end{array}$ \\
\hline 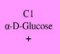 & 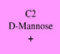 & 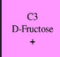 & 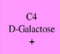 & 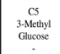 & 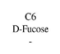 & 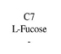 & $\begin{array}{l}c 8 \\
\text { LRenumber }\end{array}$ & 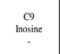 & 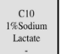 & 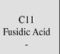 & 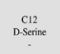 \\
\hline $\begin{array}{c}\text { DIt } \\
\text { D.sotatal }\end{array}$ & 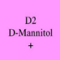 & 吕s & 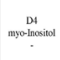 & $\begin{array}{c}\text { Ds } \\
\text { chyseral }\end{array}$ & 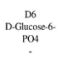 & 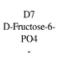 & 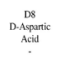 & $\begin{array}{c}\text { Do } \\
\text { DSsemina }\end{array}$ & 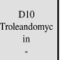 & 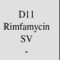 & 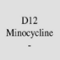 \\
\hline 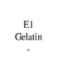 & 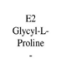 & \begin{tabular}{|l} 
Es \\
LAllume
\end{tabular} & Lergine & 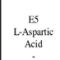 & 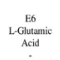 & $\begin{array}{l}\mathrm{g} 7 \\
\text { Lethidide }\end{array}$ & 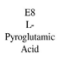 & 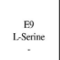 & 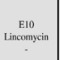 & 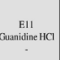 & 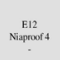 \\
\hline 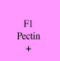 & 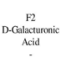 & 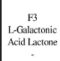 & 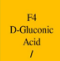 & 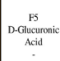 & 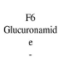 & \begin{tabular}{c} 
Mucickidid \\
\hdashline
\end{tabular} & 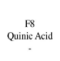 & 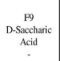 & 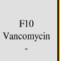 & 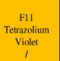 & 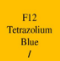 \\
\hline 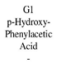 & 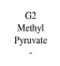 & 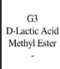 & 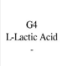 & 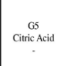 & 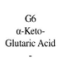 & 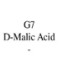 & 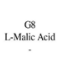 & 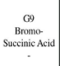 & 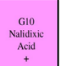 & 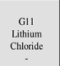 & 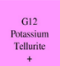 \\
\hline 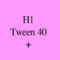 & 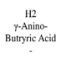 & 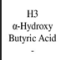 & 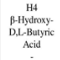 & 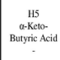 & 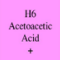 & 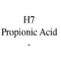 & 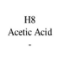 & 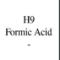 & 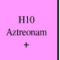 & 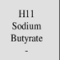 & 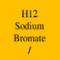 \\
\hline
\end{tabular}

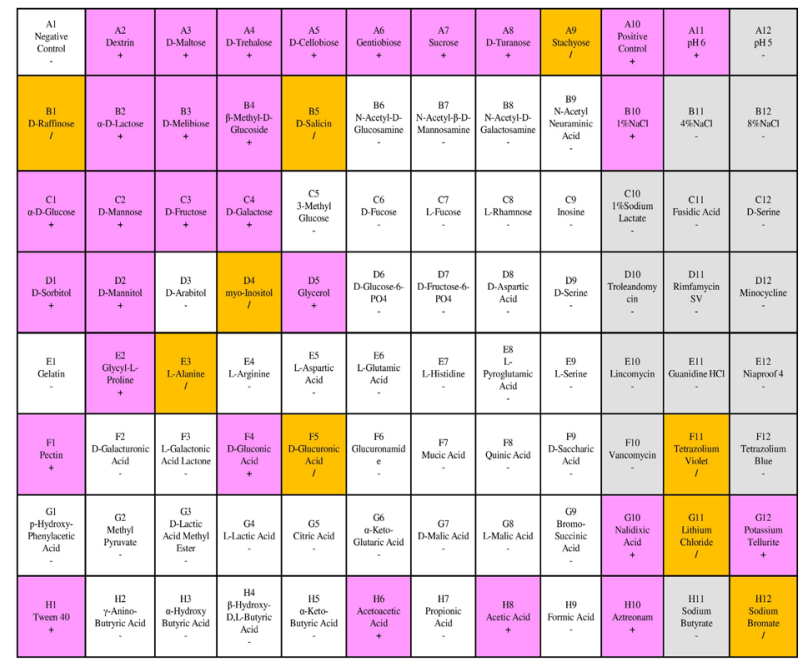

Figure 6. Interpretation of metabolic profile $\mathrm{Cmm}$ PD223 optical reading (left), spectrophotometric reading (right) + = positive reaction, lilac color; - = negative reaction, no color; / = doubtful reaction, yellow color

\begin{tabular}{|c|c|c|c|c|c|c|c|c|c|c|c|}
\hline 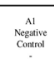 & $\underset{\substack{A 2 \\
\text { Durum }}}{i}$ & 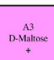 & 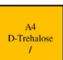 & $\begin{array}{c}\text { D.als } \\
\text { Decom } \\
+\end{array}$ & 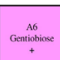 & 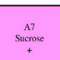 & 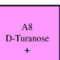 & suthoee & 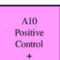 & 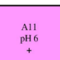 & ${ }_{\substack{A 12 \\
\text { plis }}}$ \\
\hline 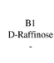 & 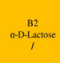 & 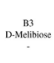 & 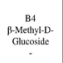 & 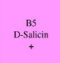 & 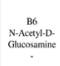 & 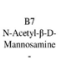 & 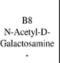 & 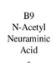 & 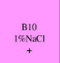 & 焉 & 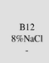 \\
\hline acilumer & 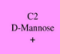 & 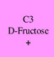 & 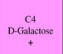 & 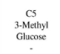 & 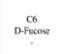 & Lheover & $\begin{array}{c}\mathrm{L} \\
\text { LRhanesese }\end{array}$ & 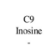 & 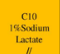 & Fialds. & 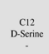 \\
\hline 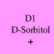 & center & 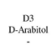 & 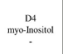 & 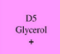 & 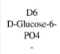 & 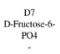 & 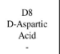 & 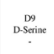 & 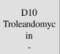 & 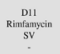 & 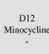 \\
\hline 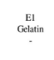 & 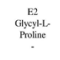 & 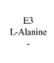 & 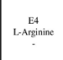 & 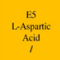 & Lothenic & L.M & 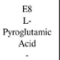 & 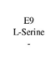 & 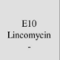 & 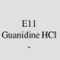 & 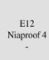 \\
\hline 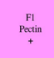 & 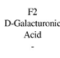 & 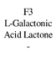 & 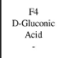 & 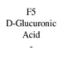 & 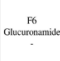 & Murie.sid & 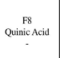 & 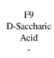 & 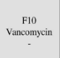 & 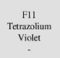 & 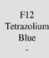 \\
\hline 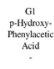 & 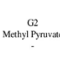 & 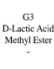 & Lexisin & ${ }^{6}$ & 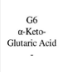 & D.W.Gle & Lavalex & 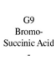 & Wilio & 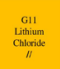 & 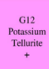 \\
\hline , & 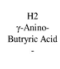 & 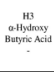 & 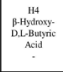 & 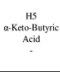 & 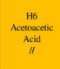 & 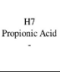 & 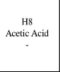 & 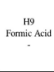 & 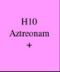 & 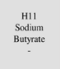 & 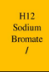 \\
\hline
\end{tabular}

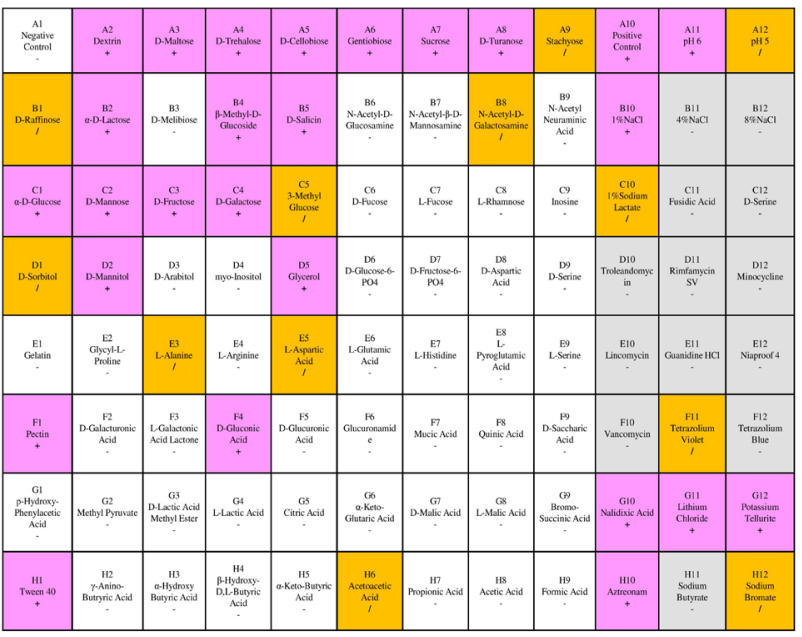

Figure 7. Interpretation of metabolic profile $\mathrm{Cmm} \mathrm{19-3857}$ optical reading (left), spectrophotometric reading (right)

+ = positive reaction, lilac color; - = negative reaction, no color; / = doubtful reaction, yellow color

pared with the reaction in well A10 which is the positive Control reaction. The positive control color is lilac. Therefore, the reactions in the wells in columns 10-12 which give a lilac color are considered positive for growth and indicate a lack of sensitivity to the respective chemical inhibitor. The wells that were not stained are considered negative for growth and indicate a significant sensitivity to the chemical inhibitor.

The metabolic profiles of the reference controls used ( $\mathrm{Cmm}$ Ncppb2979 and Cmm PD223) and the interpretation of the color reactions are shown in Figures 5-6 and for the used strains $(\mathrm{Cmm}$ 19-
3857, Cmm 19-3861, Cmm 19-4326, Cmm 194327, Cmm 19-4819) are noted in Figures 7-11.

Comparing the strains of Clavibacter michiganensis subsp. michiganensis taken in the study we can conclude that all strains use the carbon sources of the following compounds: $\alpha$-D-Glucose, Dextrin, D-Mannose, D-Mannitol, D-Fructose, D-Galactose and Sucrose. They does not use carbon sources from: p-Hydroxy-Phenylacetic Acid, Glycyl-LProline, D-Galacturonic, Methyl Pyruvate, $\gamma$-AninoButryric Acid, D-Arabitol, L-Galactonic Acid Lactone, D-Lactic Acid Methyl Ester, $\alpha$-Hydroxy Butyric Acid, L-Arginine, L-Lactic Acid, $\beta$-Hydroxy- 


\begin{tabular}{|c|c|c|c|c|c|c|c|c|c|c|c|}
\hline 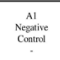 & 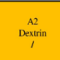 & D.s. & D. Itrathox & Dactelibix & Cancichiber & situge & $\begin{array}{l}\text { As } \\
\text { D. Thumex }\end{array}$ & $\begin{array}{c}\text { son } \\
\text { Suldyos } \\
\end{array}$ & 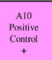 & 吾11 & $\begin{array}{c}112 \\
\text { p1ls } \\
\text { plis }\end{array}$ \\
\hline 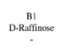 & 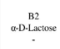 & 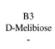 & 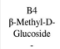 & 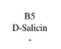 & 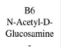 & 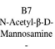 & 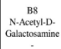 & 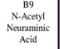 & 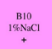 & 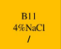 & 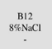 \\
\hline 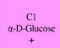 & D.inumes & 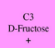 & D. Calataroce & 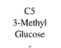 & 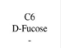 & 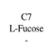 & 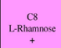 & 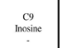 & 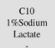 & $\begin{array}{l}\text { Fill } \\
\text { Fusidte }\end{array}$ & $\begin{array}{c}\text { C12 } \\
\text { D.sinin }\end{array}$ \\
\hline D.st & 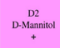 & 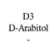 & 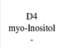 & 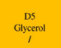 & 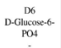 & 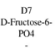 & 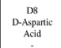 & 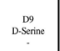 & 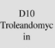 & 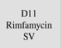 & 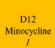 \\
\hline$\substack{\mathrm{El} \\
\text { chlutin }}$ & 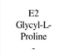 & cha & 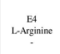 & 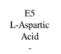 & 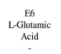 & 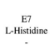 & 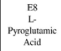 & 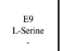 & 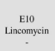 & 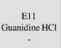 & 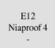 \\
\hline 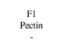 & 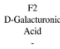 & 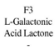 & 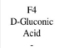 & 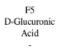 & 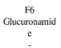 & 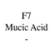 & 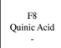 & 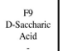 & $\begin{array}{l}\text { vino } \\
\text { vamexpin }\end{array}$ & 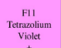 & 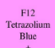 \\
\hline 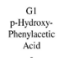 & 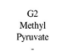 & 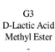 & 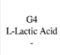 & 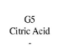 & 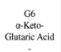 & 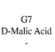 & 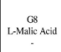 & 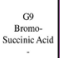 & 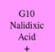 & 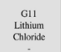 & 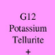 \\
\hline$\underset{\substack{\text { Hut } \\
\text { Thes to }}}{\text { to }}$ & 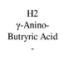 & 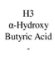 & 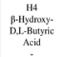 & 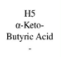 & 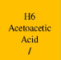 & 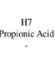 & \begin{tabular}{|c|c|} 
He \\
Anctiosid
\end{tabular} & 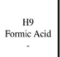 & 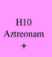 & 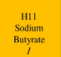 & 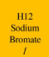 \\
\hline
\end{tabular}

\begin{tabular}{|c|c|c|c|c|c|c|c|c|c|c|c|}
\hline 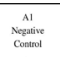 & 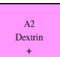 & 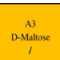 & 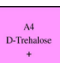 & 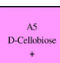 & 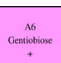 & 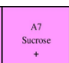 & $\begin{array}{l}\text { As } \\
\text { D-Trumese } \\
1\end{array}$ & $\begin{array}{l}\text { sulpow } \\
\text { sulpowe }\end{array}$ & 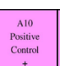 & 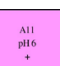 & 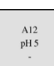 \\
\hline 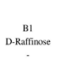 & 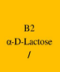 & 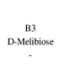 & 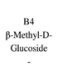 & $\begin{array}{c}\text { gs } \\
\text { D.suting } \\
1\end{array}$ & 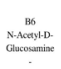 & 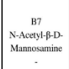 & 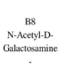 & 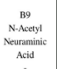 & 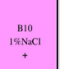 & $\begin{array}{c}\text { But } \\
\text { taxcl }\end{array}$ & $\begin{array}{c}\text { sing } \\
\text { saticac }\end{array}$ \\
\hline 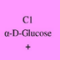 & D.wamen & Drfinuter & 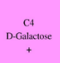 & 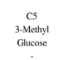 & D. & $\begin{array}{c}c \\
\text { Lheore }\end{array}$ & LRhermoxe & 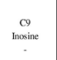 & 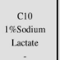 & $\begin{array}{l}\text { Fill } \\
\text { Fistit hat }\end{array}$ & 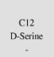 \\
\hline 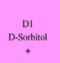 & 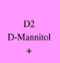 & 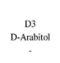 & 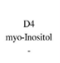 & 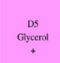 & 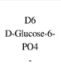 & 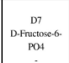 & 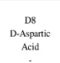 & 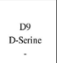 & 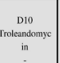 & 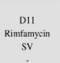 & minox) \\
\hline 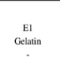 & 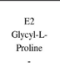 & Lather & therghe & 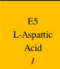 & 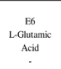 & inturate & 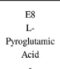 & Lent & 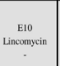 & 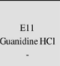 & 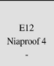 \\
\hline 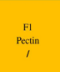 & 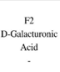 & 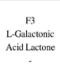 & 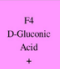 & 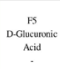 & 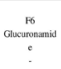 & 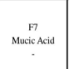 & 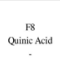 & 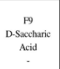 & 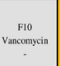 & 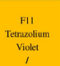 & 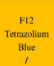 \\
\hline 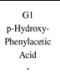 & 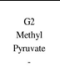 & 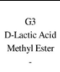 & thentide Sid & 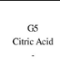 & 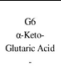 & 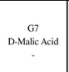 & Lishich Sid & 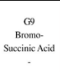 & 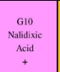 & 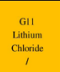 & 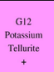 \\
\hline 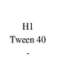 & 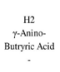 & 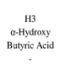 & 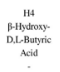 & 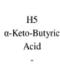 & 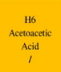 & 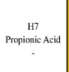 & 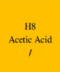 & 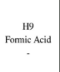 & 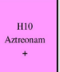 & 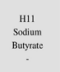 & 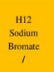 \\
\hline
\end{tabular}

Figure 8. Interpretation of metabolic profile $\mathrm{Cmm}$ 19-3861 - optical reading optical reading (left), spectrophotometric reading (right)

$+=$ positive reaction, lilac color; - = negative reaction, no color; / = doubtful reaction, yellow color

\begin{tabular}{|c|c|c|c|c|c|c|c|c|c|c|c|}
\hline 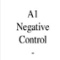 & $\begin{array}{c}M^{2} \\
\text { Dexing }\end{array}$ & 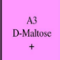 & 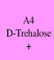 & 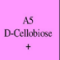 & 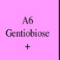 & $\begin{array}{l}\mathrm{A} 7 \mathrm{~T} \\
\text { suluse } \\
+\end{array}$ & $\begin{array}{c}\text { A8 } \\
\text { D.Thumex } \\
+\end{array}$ & 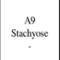 & 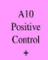 & 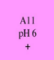 & $\mathrm{Al1}_{\mathrm{AHS}}$ \\
\hline DRatrines & 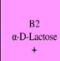 & 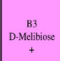 & 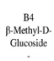 & 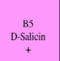 & 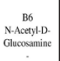 & 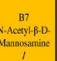 & 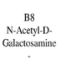 & 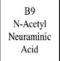 & 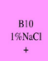 & 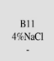 & 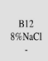 \\
\hline 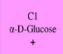 & 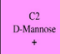 & 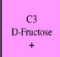 & 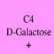 & 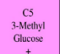 & 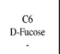 & Lenose & 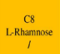 & 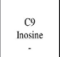 & 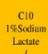 & 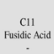 & 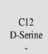 \\
\hline D.stortial & 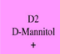 & 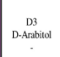 & 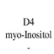 & 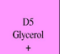 & 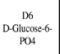 & 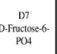 & 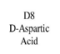 & $\begin{array}{c}\mathrm{D} \\
\mathrm{D}-\mathrm{s} \text { - sine }\end{array}$ & 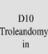 & 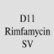 & 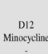 \\
\hline 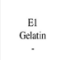 & 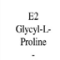 & isterine & Lhe & 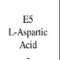 & 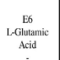 & 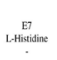 & 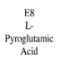 & $\begin{array}{l}\mathrm{Eg} \\
\text { LStevin }\end{array}$ & $\begin{array}{l}\text { Litio } \\
\text { Linnopyin }\end{array}$ & 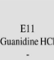 & 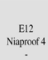 \\
\hline 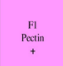 & 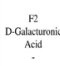 & 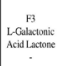 & 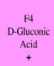 & 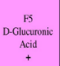 & 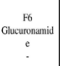 & 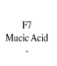 & 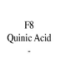 & 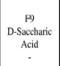 & 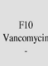 & 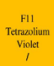 & 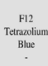 \\
\hline 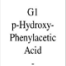 & 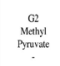 & 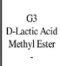 & $\begin{array}{l}\text { thatitis } \\
\text { the }\end{array}$ & 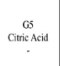 & 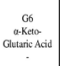 & 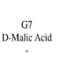 & 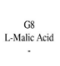 & 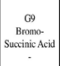 & 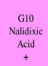 & 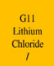 & 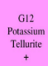 \\
\hline Trom to to & 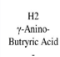 & 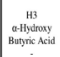 & 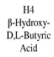 & 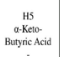 & 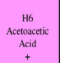 & 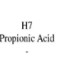 & 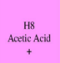 & $\begin{array}{ll}\text { Hog } \\
\text { Fomitid }\end{array}$ & $\begin{array}{c}\text { Hilo } \\
\text { suruotam } \\
+\end{array}$ & 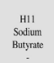 & 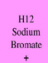 \\
\hline
\end{tabular}

\begin{tabular}{|c|c|c|c|c|c|c|c|c|c|c|c|}
\hline 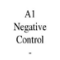 & 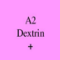 & $\begin{array}{c}B^{3} \\
\text { D.xulas } \\
+\end{array}$ & 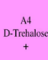 & DCchidowes & 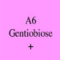 & 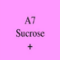 & $\begin{array}{c}\text { As } \\
\text { D.rumane } \\
+\end{array}$ & 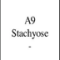 & 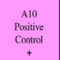 & $\underset{\substack{111 \\
\text { pHt } \\
+}}{11}$ & ${ }_{\mathrm{AH}}^{\mathrm{A12}}$ \\
\hline Dheffine & 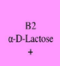 & 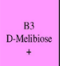 & 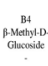 & 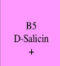 & 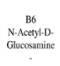 & 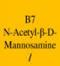 & 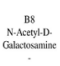 & 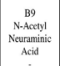 & 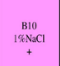 & 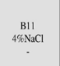 & 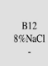 \\
\hline 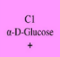 & $\underset{\substack{C_{2}^{2} \\
\text { D.Mmimace } \\
+}}{2}$ & 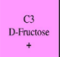 & 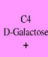 & 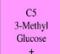 & $\underset{\substack{16 \\
\text { Drhexe }}}{ }$ & 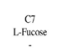 & 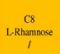 & $\underset{\substack{\text { masine } \\
\text { con }}}{ }$ & 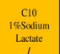 & 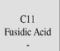 & 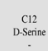 \\
\hline 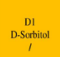 & $\begin{array}{c}\mathrm{D}^{2} \\
\text { D.M-Mumatal } \\
+\end{array}$ & 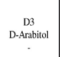 & 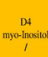 & 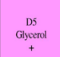 & 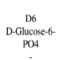 & 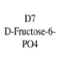 & 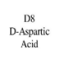 & 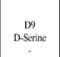 & 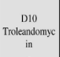 & 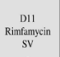 & 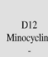 \\
\hline 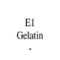 & 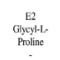 & 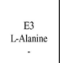 & $\begin{array}{l}\text { Let } \\
\text { Legzine }\end{array}$ & 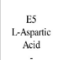 & 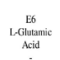 & Lithidine & 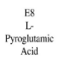 & \begin{tabular}{|c|c|c|} 
Eg \\
L-strine
\end{tabular} & Linemgen & 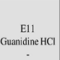 & 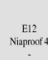 \\
\hline 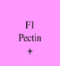 & 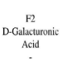 & 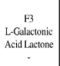 & 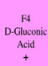 & 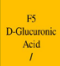 & Catronanici & 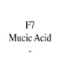 & Qininchedid & 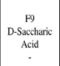 & 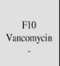 & 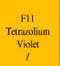 & 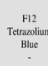 \\
\hline 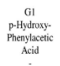 & 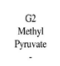 & 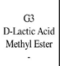 & 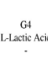 & 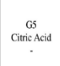 & 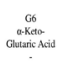 & 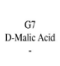 & LMhilicisid & 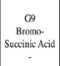 & 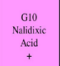 & 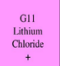 & 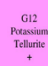 \\
\hline 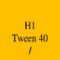 & 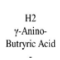 & 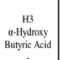 & 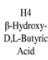 & 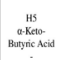 & 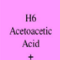 & lisktid & 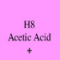 & $\begin{array}{ll}\text { Fomi } \\
\text { Fomidid }\end{array}$ & $\begin{array}{c}\text { Allo } \\
\text { Autroum } \\
+\end{array}$ & 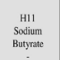 & 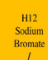 \\
\hline
\end{tabular}

Figure 9. Interpretation of metabolic profile $\mathrm{Cmm}$ 19-4326 optical reading (left), spectrophotometric reading (right) $+=$ positive reaction, lilac color; - = negative reaction, no color; / = doubtful reaction, yellow color

D,L-Butyric Acid, D-Glucuronic Acid, N-Acetyl-DGlucosamine, D-Fucose, Glucuronamide, L-Fucose, L-Histidine, Mucic Acid, D-Malic Acid, D-Aspartic Acid, L-Pyroglutamic Acid, Quinic Acid, N-Acetyl Neuraminic Acid, D-Serine, D-Saccharic Acid and Formic Acid.

With regard to chemical sensitivity tests, it was found that these strains were positive for growth in the presence of: $1 \% \mathrm{NaCl}$, Nalidixic Acid, Aztreonam, Potassium Tellurite and $\mathrm{pH}$ 6. Instead, they were negative for growth and showed sensitivity to: Troleandomycin, Lincomycin, Vanco- mycin, Fusidic Acid, Rimfamycin SV, Guanidine $\mathrm{HCl}, \mathrm{D}$-Serine, Minocycline and Niaproof 4.

Although the strain in the database metabolizes compounds: Gelatin, L-Aspartic Acid, Citric Acid, $\alpha$-Keto-Butyric Acid, D-Glucose-6-P04, L-Glutamic Acid, $\alpha$-Keto-Glutaric Acid, D-Fructose6-P04, Propionic Acid, L-Malic Acid, Inosine, L-Serine and Bromo-Succinic Acid, none of the characterized strains utilize the carbon sources of these compounds. Also, although the strain of Clavibacter michiganensis subsp. michiganensis from the database growth in the presence of 


\begin{tabular}{|c|c|c|c|c|c|c|c|c|c|c|c|}
\hline 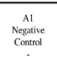 & $\begin{array}{c}\text { A2 } \\
\text { Dexulin }\end{array}$ & D.AB & 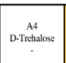 & 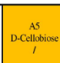 & Canibione & $\begin{array}{l}\text { A A } \\
\text { suavex } \\
+\end{array}$ & Af As & satpouc & 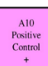 & $\begin{array}{l}\text { cult } \\
\text { pHt } \\
+ \\
+\end{array}$ & 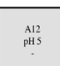 \\
\hline $\begin{array}{c}\text { But } \\
\text { Dr-ufitiox }\end{array}$ & & 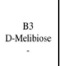 & 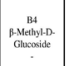 & 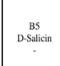 & 象 & 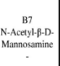 & 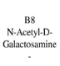 & 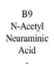 & 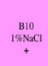 & 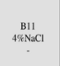 & 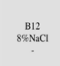 \\
\hline 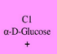 & 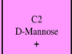 & $\begin{array}{c}\text { D. } \\
\text { D.frcinose } \\
+\end{array}$ & 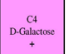 & 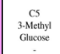 & 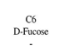 & Lefrose & Letermex & 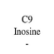 & 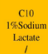 & 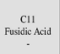 & 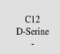 \\
\hline$\underset{\substack{\text { DI } \\
\text { D.scritiol }}}{1}$ & 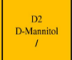 & D.Anthital & 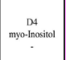 & $\begin{array}{c}\text { Ds } \\
\text { copsest } \\
+\end{array}$ & 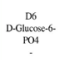 & 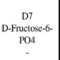 & 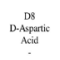 & 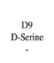 & 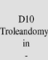 & 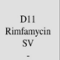 & 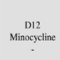 \\
\hline $\begin{array}{c}\text { El } \\
\text { cotutin }\end{array}$ & 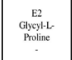 & $\begin{array}{l}\text { IB } \\
\text { LAfluine }\end{array}$ & 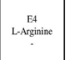 & 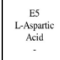 & 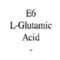 & Lithitione & 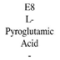 & 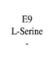 & $\begin{array}{c}\text { Eilo } \\
\text { Linnoursin }\end{array}$ & 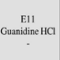 & 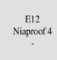 \\
\hline$\underset{\substack{\mathrm{fr} \\
\text { prxin } \\
+}}{+}$ & 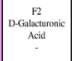 & 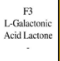 & 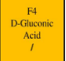 & 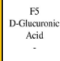 & 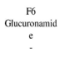 & 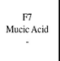 & 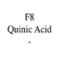 & 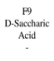 & $\begin{array}{c}\text { Fil } \\
\text { Vanomanci }\end{array}$ & 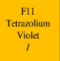 & 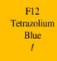 \\
\hline 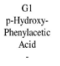 & 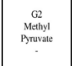 & 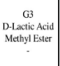 & 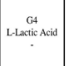 & 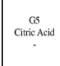 & 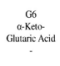 & 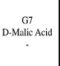 & 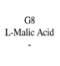 & 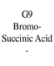 & 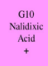 & 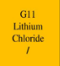 & 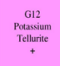 \\
\hline 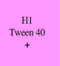 & 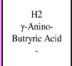 & 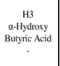 & 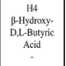 & 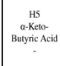 & chost & Propinicica & 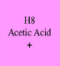 & 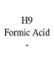 & 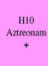 & 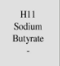 & 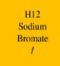 \\
\hline
\end{tabular}

\begin{tabular}{|c|c|c|c|c|c|c|c|c|c|c|c|}
\hline 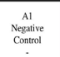 & 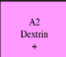 & 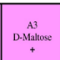 & D. It & Distitioe & 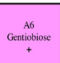 & $\begin{array}{c}\text { Sune } \\
\text { suane }\end{array}$ & 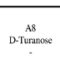 & Sulthox & 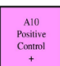 & 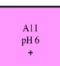 & ${ }^{\mathrm{A} 12}$ \\
\hline atsine & 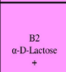 & $\begin{array}{l}\text { Bh3 } \\
\text { D-Mcthox }\end{array}$ & 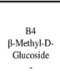 & $\begin{array}{c}\text { Bs } \\
\text { D.sildin }\end{array}$ & 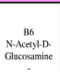 & 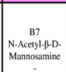 & 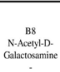 & 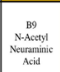 & 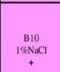 & 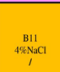 & 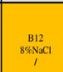 \\
\hline 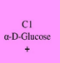 & 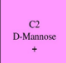 & Defmuree & 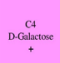 & 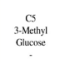 & 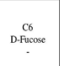 & 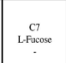 & L.Rhansese & 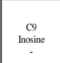 & 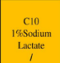 & 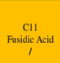 & 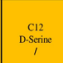 \\
\hline 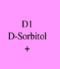 & 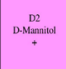 & 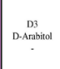 & 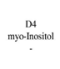 & 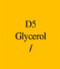 & 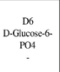 & 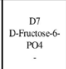 & 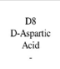 & 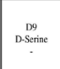 & 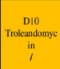 & $\begin{array}{l}\text { Din } \\
\text { Rimflandin } \\
\text { sy }\end{array}$ & (x) \\
\hline 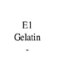 & 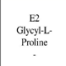 & 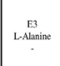 & 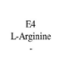 & 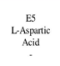 & 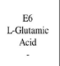 & Lithine & 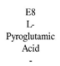 & 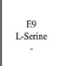 & 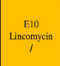 & 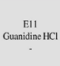 & 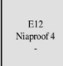 \\
\hline$\underset{\substack{\text { frecuin } \\
\text { fin }}}{\mathrm{F}}$ & 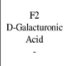 & 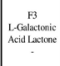 & 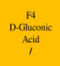 & 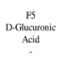 & $\begin{array}{c}\text { Gerfonid } \\
\vdots \\
\vdots\end{array}$ & Marickida & Orithith & 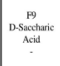 & 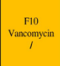 & 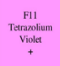 & 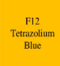 \\
\hline 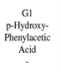 & 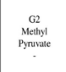 & 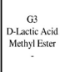 & 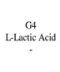 & 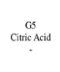 & 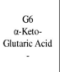 & 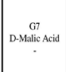 & Livalictidid & 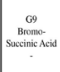 & 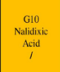 & 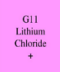 & 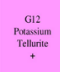 \\
\hline 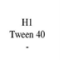 & 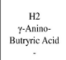 & 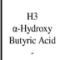 & 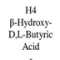 & 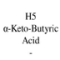 & 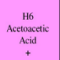 & 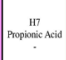 & 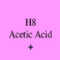 & Fromiestadid & $\begin{array}{c}\text { Allo } \\
\text { Anteram }\end{array}$ & 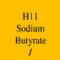 & 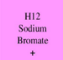 \\
\hline
\end{tabular}

Figure 10. Interpretation of metabolic profile $\mathrm{Cmm}$ 19-4327 optical reading (left), spectrophotometric reading (right)

+ = positive reaction, lilac color; - = negative reaction, no color; / = doubtful reaction, yellow color
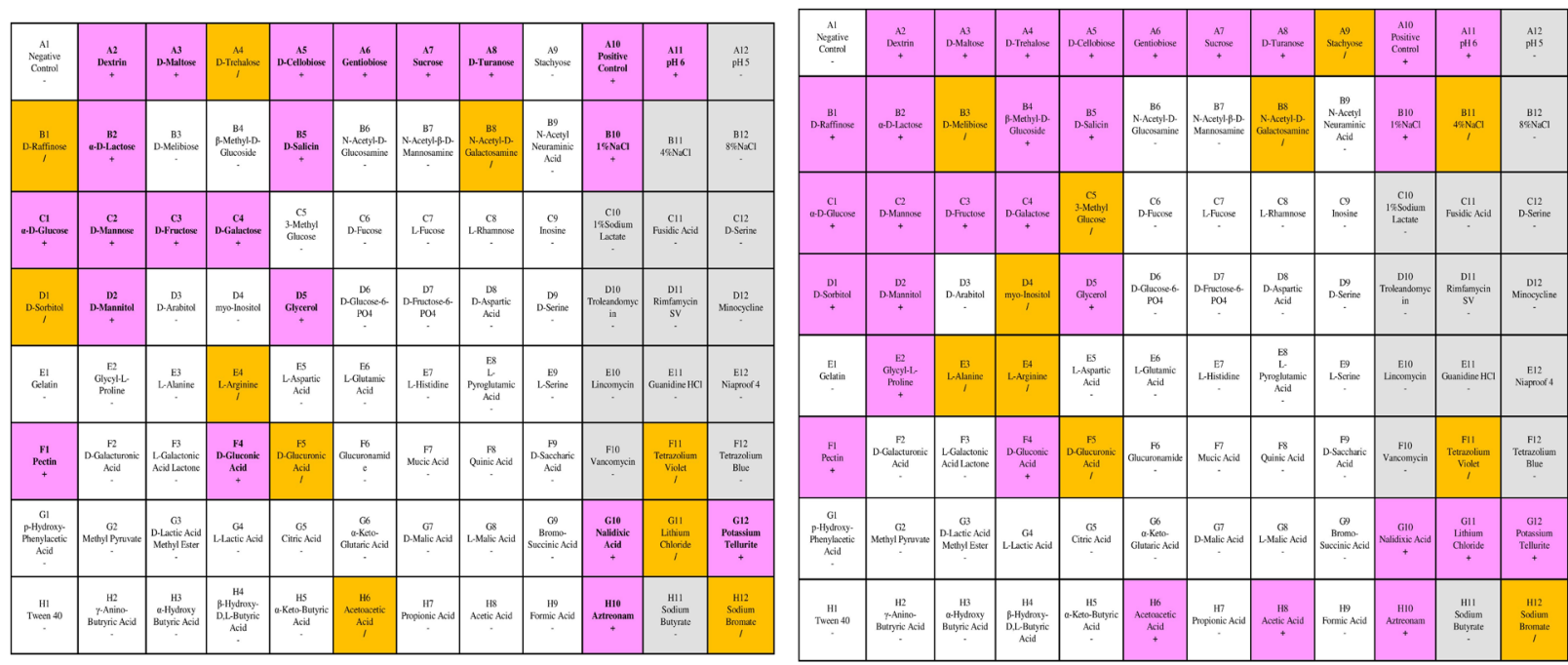

Figure 11. Interpretation of metabolic profile of $\mathrm{Cmm} 19-4819$ optical reading (left), spectrophotometric reading (right)

+ = positive reaction, lilac color; - = negative reaction, no color; / = doubtful reaction, yellow color

Sodium Butyrate and $8 \% \mathrm{NaCl}$, all strains studied did not develop in the presence of these compounds.

The rest of the reactions were varied, depending on the strain, having either a positive or a negative result, without a uniformity in all the strains, regarding the response to a certain type of reaction. There were also wells on the microplates which had an uncertain result, which was interpreted as a limit reaction, which could not be classified as positive or negative, or which showed a reaction mismatch with the reaction in the database for Clavibacter michiganensis subsp. michiganensis. The strains studied had more intense or weaker reactions compared to the strain in the database. Also, between the optical and spectrophotometric readings there were slight differences, without fundamentally altering the metabolic profile of the strain analyzed.

By summing the number of positive reactions, the metabolic diversity of the strains studied was calculated. Thus, in this case the number of positive reactions varied between $12-28$ positive reac- 


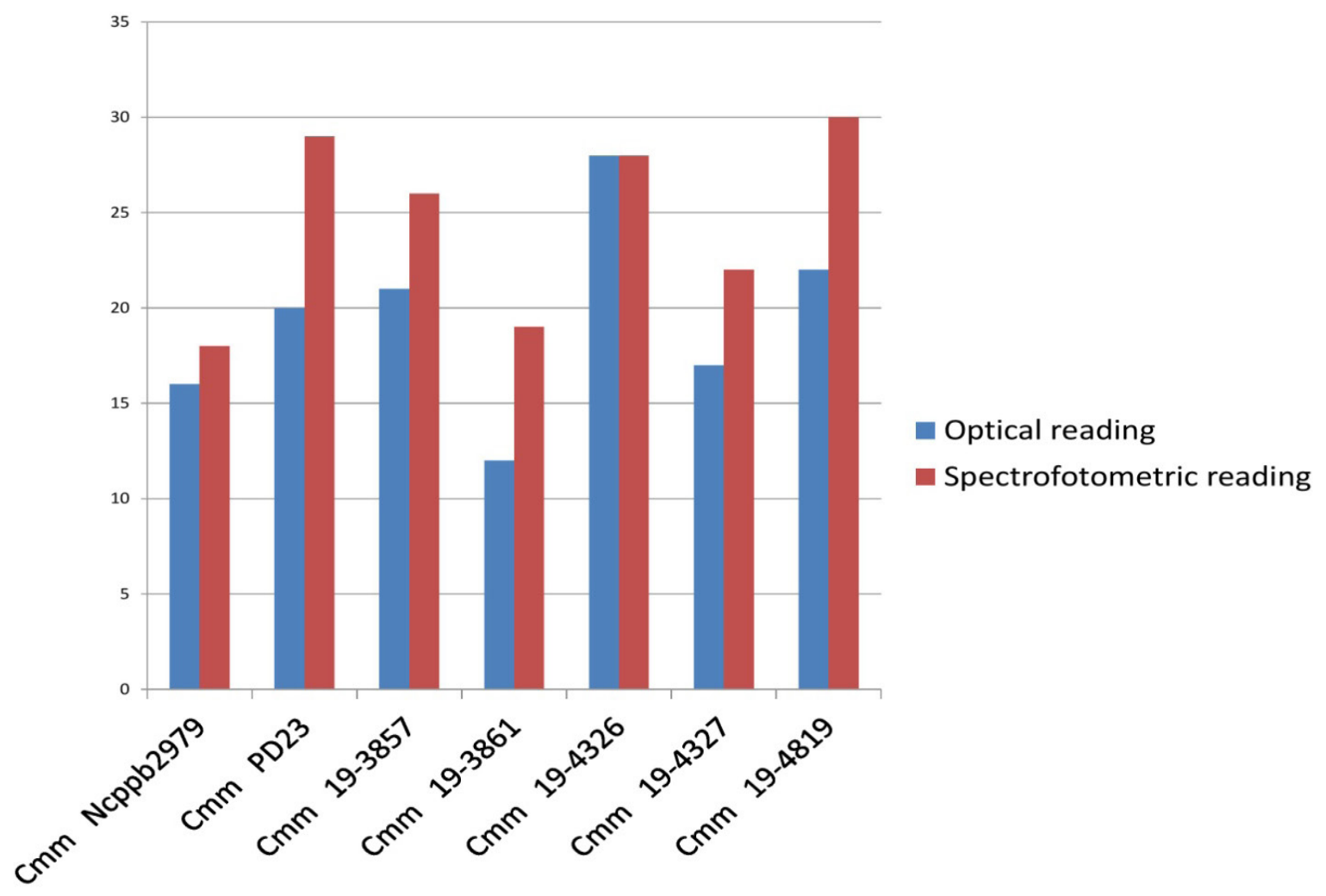

Figure 12. Metabolic diversity of Clavibacter michiganensis subsp. michiganensis

tions for optical readings and between $18-39$ positive reactions for spectrophotometric readings (Fig. 12).

\section{Conclusion}

In this study there were identified strains of Clavibacter michiganensis subsp. michiganensis only up to the level of the genus and species (Clavibacter michiganensis) not to the level of the subspecies.

The existence of the differences between the database of the Biolog microbial identification system and the strains taken in the study did not essentially change the classification of the strains at the level of genus and species but did modify or did not allow their exact classification at the subspecies level.

Only one strain was erroneously classified at the subspecies level, due to the similarity of the metabolic profile with another organism of the Clavibacter genus. It was framed only for optical readings, not spectrophotometric ones, in the subspecies sepedonicus.

The visual analysis allowed only to establish that the cells existing in the microplates were alive and had an active metabolism without allowing their biochemical characterization. Optical and spectrophotometric analysis (performed at two wavelengths) allowed us to characterize biochemically the strains of Clavibacter michiganensis subsp. michiganensis taken in the study. Following this characterization it was found that the analyzed strains had metabolic diversity, because they metabolized different substrates, using different carbon sources.

\section{References}

1. Bonkowski J, Joseph B, Bayo D (2015). Clavibacter michiganensis subsp. michiganensis. https://wiki.bugwood. org/Clavibacter_michiganensis_subsp._michiganensis.

2. Jahr H, Dreier J, Meletzus D, Bahro R, Eichenlaub R (2000). The endo-b-1,4-glucanase CelA of Clavibacter michiganensis subsp. michiganensis is a pathogenicity determinant required for induction of bacterial wilt of tomato. Molecular Plant-Microbe Interactions, 13(7): 703-714.

3. Sen Y, Van der Wolf J, Visser RGF, Van Heusden S (2015). Bacterial canker of tomato: current knowledge of detection, management, resistance, and interactions. Plant Disease, 99(1): 4-13.

4. Severin V, Iliescu CH (2006). Bolile bacteriene ale plantelor. Ed. Geea București. 49, 158-163. 
5. Singh G, Bharat NK (2017). Studies on bacterial canker (Clavibacter michiganensis subsp. michiganenesis) of tomato (Solanum lycopersicum). International Journal of Current Microbiology and Applied Sciences, 6(9): 317323.
6. User guide - Identification system Biolog.

7. EPPO Global Database. Clavibacter michiganensis subsp. michiganensis. https://gd.eppo.int/taxon/CORBMI/hosts. 